ten or more medications, even after exclusion of IDU indicators from the total number of medications prescribed.

Conclusions The total number of medications and IDU indicators are associated with ADE among older people. Although IDU indicators were positively associated with ADE, they lacked to fully explain the graded association with increasing numbers of medications prescribed. Physicians, but also patients need to be aware of this increased likelihood of serious ADE by low and increasing numbers of medications prescribed.

\section{EDUCATIONAL INTERVENTIONS DESIGNED TO IMPROVE MEDICATION ADMINISTRATION SAFETY}

${ }^{1}$ Marja Härkänen, ${ }^{1}$ Ari Voutilainen, ${ }^{2}$ Elina Turunen, ${ }^{1,2}$ Katri Vehviläinen-Julkunen. ${ }^{1}$ University of Eastern Finland, Finland; ${ }^{2}$ Kuopio University Hospital, Finland

10.1136/injuryprev-2016-042156.558

Background Increasing the medication administration safety is vital to improving patient safety. The aim of this study is to analyse educational interventions designed to increase the medication administration skills and safety of registered nurses.

Methods A systematic review of the literature using six databases was conducted to identify intervention studies published between January 2000 and April 2015. The quality of studies was assessed using the Effective Public Health Practice Project (EPHPP) Quality Assessment Tool. The efficacy of the interventions was determined by calculating the effect sizes and conducting a metaanalysis.

Results Fourteen intervention studies were included in this systematic review. Quality was strong only in one study and moderate in another four. The interventions differed significantly from one study to the next, and the original investigators reported significant improvement in the skills and medication safety owing to the interventions. The meta-analyses, however, revealed that the effect of the intervention was strong in only four of the studies. Those interventions were the 60-minute educational intervention (PowerPoint presentation), a blended learning programme inclusive of e-learning, wall poster and informative pamphlets, and a combination of three different interventions (classroom or selfstudy methods). In two out of four cases, the quality of the study was weak and moderate in two cases, and only one of the effective interventions was a randomised controlled trial.

Conclusions Based on the original analysis, all the interventions reviewed had a positive impact on medication administration safety. Nonetheless, these positive outcomes do not necessarily indicate effective interventions. The most effective interventions in this review were methodologically dissimilar. Studies should be assessed for their quality and a meta-analysis performed when searching for the best, effective and highest quality educational interventions.

\section{EXAMINING THE RELATIONSHIP BETWEEN EDUCATION AND ALCOHOL-ATTRIBUTABLE NON-FATAL INJURY RISK IN BRAZIL}

${ }^{1}$ Deena El-Gabri, ${ }^{1}$ Nicole Toomey, ${ }^{1,2}$ João Ricardo Vissoci, ${ }^{1,3}$ Catherine Staton. ' Duke Global Health Institute; ${ }^{2}$ Faculdade Ingá; ${ }^{3}$ Duke University Medical Centre

10.1136/injuryprev-2016-042156.559

Background In Brazil, alcohol use is a leading risk factor for injury contributing to over $8 \%$ of DALYs lost. While alcohol consumption increases with income, there is an inverse risk of alcohol-attributable mortality with socioeconomic status (SES). Education is an important measure of SES; yet its effect on risk for non-fatal injury is unclear. This project investigates education level most at risk for alcohol positive injury in Maringá, Brazil.

Methods Self-reported alcohol usage two hours prior to injury was collected during a survey on treatment-seeking behaviour following injury in Maringá, Brazil between May and September 2015. Households provided demographics, and one randomly selected household member supplied injury history. Alcohol usage prior to injury and demographics were analysed using frequencies and logistic regression. Risk ratios were calculated using primary school incomplete, the lowest risk group, as reference.

Results Of 2678 people surveyed, 797 reported injury. Only 56 (7.02\%) reported alcohol use of which $85.7 \%$ were male. Of all alcohol-attributable injured patients, 3 (5.4\%) did not complete primary school, $8(14.3 \%)$ completed primary school, 12 (21.4\%) completed secondary school, and 15 (26.8\%) completed professional school. Those who completed primary education and professional education were at similar risk for alcohol-attributable injuries. Completing primary school $(\mathrm{RR}=4.14$, $\mathrm{p}=0.032)$ and professional school $(\mathrm{RR}=3.43, \mathrm{p}=0.047)$ had the highest risk of alcohol-induced non-fatal injury.

Conclusions Alcohol use is a major risk factor for injury and is influenced by education. While our sample size is small and only includes non-fatal injuries, it mirrors the general pattern of alcohol usage seen in Brazil. We found low and high levels of education were at highest risk for alcohol-related injury. Our data suggests an indirect dependence of education on alcohol-induced injury however this relationship requires more in depth research.

\section{ALCOHOL LEVELS AND INJURY RELATED MORTALITY}

Ms Aine O'Brien, Michal Molcho. School of Health Sciences, NUI Galway, Ireland

\subsection{6/injuryprev-2016-042156.560}

Background Limited data on injury mortality were traditionally collected using death certificates. Coroners' inquest reports offer a richer source of injury mortality data; collating information from multiple sources (including police reports, witness statements, coronial autopsy and forensic pathologist post-mortem and toxicology examinations). These reports provide information on the manner and mechanism of injury, the presence of alcohol and drugs, a detailed description of injuries sustained and a narrative of the circumstances resulting in death.

Methods This study reviewed 722 coroners' inquest investigation reports of injury related death between 2006 and 2010 in the West of Ireland, including coronial and forensic pathologist postmortem and toxicology examination results. Alcohol levels were measured in blood and urine and included in toxicology results. This paper focuses on the presence of alcohol in various injury related fatalities.

Results Alcohol was detected in $54.2 \%$ of cases $(78 \%$ male and $22 \%$ female), most commonly among 0-44 year-olds (49\%). Where alcohol was present, it was most frequently at a level greater than $200 \mathrm{mg} / 100 \mathrm{ml}$. Alcohol was present in $41 \%$ of unintentional fatalities, $19 \%$ of intentional fatalities, and $40 \%$ of undetermined intent fatalities. Alcohol was detected in $40 \%$ of asphyxiated fatalities, consisting of $49.7 \%$ hanging and $41.4 \%$ of drowning fatalities. Blood alcohol levels $>200 \mathrm{mg} / 100 \mathrm{ml}$ were found in $39 \%$ of the drowning fatalities, while levels $<20 \mathrm{mg} / 100$ $\mathrm{ml}$ were detected in $30 \%$ of the hanging fatalities. 